Coeliac disease

\section{The changing face of coeliac disease}

\section{R M Beattie}

\section{Perspective on the paper by Ravikumara et al (see page 969)}

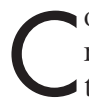
liac disease is an autoimmunemediated enteropathy triggered by cally susceptible individuals. The major predisposing genotypes are HLA-DQ2 and HLA-DQ8 found in at least $98 \%$ of patients. The widespread use of antibody screening has considerably changed the clinical spectrum of new cases seen with increasing recognition, through the testing of children with less classic symptoms and screening of children at high risk, of the varied presentation and increased prevalence of this now common condition.

Coeliac disease was previously considered to be rare, with an estimated prevalence of 1 in 2000. This was before the widespread availability of antibody screening. The prevalence of coeliac disease, based on either cross-sectional or population-based studies in Western populations, is in the order of $0.3-2 \%$, with a higher prevalence in at-risk groups. ${ }^{1-3}$ The vast majority of cases, however, remain undetected, with seropositivity in apparently healthy individuals when populations are screened (silent coeliac disease). This is commonly referred to as the coeliac iceberg. The potential disease burden if all cases are detected is huge.

Ravikumara et $a l^{4}$ have reported 21 years of prospectively held data on the mode of presentation of coeliac disease in a single centre, with increasing availability and progressively increasing sensitivity and specificity of antibody testing during that period. The number of new cases increased from 11 patients diagnosed between 1983 and 1989 to 50 between 1999 and 2004. This study shows that gastrointestinal manifestations are now less prominent at diagnosis with 1 in 4 patients diagnosed during 1999-2004 as a consequence of targeted screening. Median age at presentation shifted from 4 to 8 years during this period. The same group has previously reported an incidence of between 1 in 2500 and 1 in 3000 live births in the 1990s, ${ }^{5}$ and more recently, a prevalence among screened healthcare professionals of 1 in $166,{ }^{6}$ suggesting that the significant coeliac iceberg reported elsewhere is a major issue in the UK as well. ${ }^{7}$
There are three settings in which the diagnosis of coeliac disease should be considered: children with frank gut symptoms, children with non-gastrointestinal manifestations and asymptomatic individuals with conditions that are associated with coeliac disease. Non-gastrointestinal manifestations include dermatitis herpetiformis, reduced bone mineral density, dental enamel hypoplasia, short stature, delayed puberty, iron-deficiency anaemia not responsive to iron supplements and infertility. Conditions that are associated with coeliac disease include type 1 diabetes mellitus, immunoglobulin (Ig) A deficiency, Down's syndrome, Turner's syndrome, Williams' syndrome and firstdegree relatives of those with coeliac disease $(10 \%)$. The question as to whether all children should be screened is more difficult and controversial, ${ }^{8-10}$ with published debates on the merits of screening the general population ${ }^{11} 12$ and the population with diabetes. ${ }^{13}$

The diagnosis and benefits of treatment are well reviewed and well accepted in children with coeliac disease who are screened on the basis of symptoms. ${ }^{1-3}$ The risks of non-treatment include persistent gastrointestinal symptoms, impaired nutrition, impaired growth and pubertal development, reduced bone mineralisation leading to osteoporosis, infertility and an increased risk of gastrointestinal malignancy. The North American Society of Paediatric Gastroenterology, Hepatology and Nutrition (NASPGHAN) has just produced evidence-based consensus guidelines. ${ }^{2}$

The symptoms, however, can be nonspecific and include short stature in which coeliac serology should be a routine investigation, ${ }^{14}$ with good catch-up growth seen once the condition is diagnosed. It is more difficult in children who are picked up by either high-risk group screening or population-based screening, in whom the diagnosis can be less clear and treatment decisions more controversial, particularly if the children are, and perceive themselves as, asymptomatic. This will clearly have an effect on acceptance of the diagnosis and compliance.

It is crucial to make a definitive diagnosis because of the lifelong implications of the condition. This has recently been reviewed in the comprehensive NASPGHAN guidelines. ${ }^{2}$ The most widely used screening test is the measurement of IgA antibody to human recombinant tissue transglutaminase and serum IgA. Sensitivity and specificity approach 100\%. False positives can, however, occur. It is important to exclude IgA deficiency as a cause of false-negative serology. In such children, IgG transglutaminase antibody levels can be measured. Measurement of IgA antibody to endomysium is observer dependent and expensive. Antigliadin antibody tests are less accurate and are not advised.

It is crucial that children having coeliac disease testing are on a normal, glutencontaining diet. If children have already been started on a gluten-free diet or are eating insufficient amounts of gluten, they should be referred to a paediatric dietician and advised to reintroduce gluten in their diet for at least 3 months (preferably longer), with serial serological testing if there is a high clinical suspicion of coeliac disease and small bowel biopsy following positive serology. It is not usually adequate to return to a normal diet for just 2-3 weeks before biopsy. After a period of gluten exclusion, it may take many months for serology to turn positive. Late relapse after gluten challenge has been reported.

All children with positive serology should have small bowel biopsy before starting a gluten-free diet. Children for whom there is a high clinical suspicionfor example, faltering growth or chronic diarrhoea-should be referred for consideration of a biopsy even if their serology test is negative, as coeliac disease has been reported in this setting (rarely) and other enteropathies may be found. Children with positive serology who initially have normal biopsy results may subsequently develop mucosal abnormalities.

Diagnosis is based on a small bowel biopsy showing characteristic histological findings of partial or complete villous atrophy, crypt hyperplasia and increased intraepithelial lymphocytes with a lamina propria plasma cell infiltrate in the presence of positive serology. Biopsies are usually taken endoscopically. Multiple biopsies should be taken, as the lesion can be patchy.

The diagnosis is confirmed by complete symptom resolution on a strict gluten-free diet. Dietetic input is essential. Positive serology should revert to negative over time. If there is no decline in antibody levels after 6 months, compliance should be reviewed.

Human lymphocyte antigen typing can be considered in high-risk groups or in children in whom the diagnosis is uncertain. Coeliac disease is unlikely in those who are not HLA-DQ2 or HLADQ8 positive. 
A formal gluten challenge is rarely indicated, particularly if serology is informative and the biopsy characteristic, but this may be helpful in difficult cases, particularly if there is diagnostic uncertainty (eg, lack of clarity about the initial diagnosis, gluten exclusion with no biopsy). The challenge should be supervised by a paediatric dietician. Relapse can occur many months after the challenge.

There is very little data on the outcome of coeliac disease in children who are asymptomatic at presentation and picked up through screening, although a pragmatic presumption that the same longterm health benefits occur as in children symptomatic at diagnosis and therefore the recommendation is that all biopsy positive children should be treated. There is some evidence that children apparently asymptomatic at diagnosis have mild impairment of growth and are more likely to have symptoms (irritability, lethargy, distension and gas) than control subjects. ${ }^{15}$ It is likely, therefore, that some patients are considered asymptomatic when they are not with ill health, only being noticed in retrospect.

Type 1 diabetes has been the most widely studied with respect to high-risk screening and outcome, with the prevalence of coeliac disease in children with type 1 diabetes being around $4 \% .{ }^{16}$ There is no evidence for an improvement in diabetic control short term. The medium and longer term effects of diabetic control are also unknown; in particular, it is unclear whether treatment of coeliac disease impacts on the potential to develop other autoimmune conditions.

It is important to remember that children in high-risk groups whose serology is initially negative on screening may develop a positive serology subsequently. It is sensible to repeat testing if children at high risk develop suspicious symptoms.

The NASPGHAN recommends that screening should begin at 3 years in asymptomatic, high-risk children who have been on an adequate gluten-containing diet for at least 1 year before testing. ${ }^{2}$ There is no consensus on how often screening should be carried out. Guidance from the National Institute of Clinical Excellence (UK) recommends screening those with type 1 diabeties at diagnosis and then every 3 years. ${ }^{17}$

It is clearly necessary to have a low threshold to investigate for coeliac disease in a child with either frank or occult gut symptoms. It should be a routine part of the initial screening in children of short stature. It is crucial that the diagnosis is made correctly, and a trial of gluten exclusion in children in whom the diagnosis is suspected is not recommended. The high prevalence of coeliac disease is a major healthcare issue and is relevant to healthcare planning. We need to know the natural history of undetected coeliac disease to determine, whether we should screen the whole population or high-risk groups or only those who are symptomatic. Until these issues are resolved, we, as the team from Cardiff emphasise, must maintain a high index of suspicion for this condition so that the potential problems associated with untreated coeliac disease can be prevented.

Arch Dis Child 2006;91:955-956.

doi: 10.1136/adc. 2006.099671

Correspondence to: R M Beattie, Paediatric Medical Unit, Southampton General Hospital, Tremona Road, Southampton SO16 6YD, UK; mark.beattie@suht.swest.nhs.uk

Competing interests: None declared.

\section{REFERENCES}

1 Feighery C. Coeliac disease. BMJ 1999:319:236-9.

2 Hill ID, Dirks MH, Liptak GS, et al. Guideline for the diagnosis and treatment of coeliac disease in children: recommendations of the North American Society for Pediatric Gastroenterology, Hepatology and Nutrition. J Pediatr Gastroenterol Nutr 2005;40:1-19.

3 van Heel DA, West J. Recent advances in coeliac disease. Gut 2006;55:1037-46.

4 Ravikumara M, Tuthill DP, Jenkins HR. The changing clinical presentation of coeliac disease. Arch Dis Child 2006;91:969-71.

5 Jenkins HR, Hawkes N, Swift GL. Incidence of coeliac disease. Arch Dis Child 1998;79:198.

6 El-hadi S, Tuthill D, Lewis $E$, et al. Unrecognised coeliac disease is common in healthcare students. Arch Dis Child 2004;89:842.

7 Fasano A. Clinical presentation of celiac disease in the paediatric population. Gastroenterology 2005; 128:S68-73.

8 Young EH, Wareham NJ. Screening for coeliac disease: what evidence is required before population programmes could be considered. Arch Dis Child 2004:89:499-500.

9 Tommasini A, Not T, Kiren V, et al. Mass screening for coeliac disease using antihuman transglutaminase antibody assay. Arch Dis Child 2004;89:512-15.

10 Hoffenberg EJ. Should all children be screened for coeliac disease? Gastroenterology 2005; 128:S98-103.

11 Fasano A. European and North American populations should be screened for coeliac disease (protagonist). Gut 2003;52:168-9.

12 Kumar PJ. European and North American populations should be screened for coeliac disease (antagonist). Gut 2003:52:170-1.

13 Freemark M, Levitsky LL. Screening for coeliac disease in children with type 1 diabetes: two views of the controversy. Diabetes Care 2003;26: 1932-9.

14 van Rijn JC, Grote FK, Oostdiik W, et al. Short stature and the probability of coeliac disease, in the absence of gastrointestinal symptoms. Arch Dis Child 2004;89:882-3.

15 Hoffenberg EJ, Emery LM, Barriga KJ, et al. Clinical features of children with screen identified evidence of coeliac disease. Pediatrics 2004; 113:1254-9.

16 Holmes GK. Screening for coeliac disease in type 1 diabetes mellitus. Arch Dis Child 2002;87:495-9.

17 National Institute for Clinical Health and Clinical Excellence. Diagnosis and management of type 1 diabetes in infants, young people and adults. Birmingham, UK: NICE, 2004.

\section{One hundred years of telemedicine: does this new technology have a place in paediatrics?}

\section{E M Strehle, N Shabde}

\section{0 years of telemedicine}

A lthough hard to believe, this year we celebrate the looth anniversary of telemedicine. The term telemedicine was coined in the 1970s by the American Thomas Bird and, literally translated, means "healing at a distance" (from Latin "medicus" and Greek "tele"). However, the origins of this evolving technology date back to the early 20th century, when Willem
Einthoven, a Dutch physiologist, developed the first electrocardiograph in his laboratory in Leiden. With the use of a string galvanometer and telephone wires, he recorded the electrical cardiac signals of patients in a hospital $1 \frac{1}{2} \mathrm{~km}$ away. He stated: "Where there is a link, actual and figurative, between laboratory and hospital, and collaboration between physiologist and clinician, each remaining master in his territory, there one may fruitfully utilize these new electrical methods of research". Einthoven's electrocardiograph was very large but over the years was transformed into a mobile or even portable monitoring device. Nevertheless, he can be regarded as the first clinician scientist to develop and systematically apply a technique that is very similar to telemedicine in the modern sense. 